\title{
AN ANALOGUE OF THE MOSTOW-MARGULIS RIGIDITY THEOREMS FOR ERGODIC ACTIONS OF SEMISIMPLE LIE GROUPS ${ }^{1}$
}

\author{
BY ROBERT J. ZIMMER ${ }^{2}$
}

In this paper we announce an analogue of the rigidity theorems for lattices in semisimple Lie groups of Mostow and Margulis in the context of ergodic actions of semisimple Lie groups and ergodic foliations by symmetric spaces.

If $G, G^{\prime}$ are locally compact (second countable) groups acting ergodically on standard measure spaces $S, S^{\prime}$ respectively, the actions are called orbit equivalent if, possibly after discarding invariant null sets, there is a measure class preserving Borel bijection $S \rightarrow S^{\prime}$ that takes orbits onto orbits. For example, if $G=G^{\prime}$ and the actions are conjugate (i.e., the map $S \rightarrow S^{\prime}$ is a $G$-map), or more generally, automorphically conjugate (i.e., conjugate modulo an automorphism of the group) then the actions are clearly orbit equivalent. However, orbit equivalence is in general a far weaker notion. For example, if $G$ and $G^{\prime}$ are discrete amenable groups and the measures are finite and invariant (and properly ergodic, i.e., not transitive modulo a null set), then the actions will be orbit equivalent [1], [2], [7]. The same result is true for essentially free (i.e., almost all stabilizers are trivial) actions of continuous amenable groups. Our main result is Theorem A which asserts that the situation for semisimple Lie group actions is very different. This result has been independently conjectured by $\mathbf{A}$. Connes. An ergodic action of a semisimple Lie group is called irreducible if the restriction to every noncentral normal subgroup is still ergodic.

THEOREM A. Let $G, G^{\prime}$ be connected seimsimple Lie groups with finite center and no compact factors, and suppose the real rank of $G$ is at least 2. Suppose $S, S^{\prime}$ are essentially free, irreducible ergodic $G, G^{\prime}$ spaces respectively with finite invariant measure. If the actions are orbit equivalent then $G$ and $G^{\prime}$ are locally isomorphic. If $G$ and $G^{\prime}$ have trivial center, then $G=G^{\prime}$ and the actions are automorphically conjugate.

Received by the editors August 15, 1979.

AMS (MOS) subject classifications (1970). Primary 22D40, 22E40, 28A65, 57D30; Secondary $46 \mathrm{~L} 10$.

1 Partially supported by NSF grant NSF MCS76-06626.

${ }^{2}$ Sloan Foundation Fellow. 
While the Mostow-Margulis rigidity theorems [4], [6] assert that an isomorphism of lattice subgroups extends to an isomorphism of the Lie group, Theorem A can be interpreted as asserting that an isomorphism of certain virtual subgroups [3] extends to an isomorphism of the groups.

As a consequence, we deduce the following.

THEOREM B. Let $\Gamma \subset G$ and $\Gamma^{\prime} \subset G^{\prime}$ be lattices in connected simple Lie groups with finite center and suppose the real rank of $G$ is at least 2. If $S$ and $S^{\prime}$ are essentially free ergodic $\Gamma$ and $\Gamma^{\prime}$-spaces respectively with finite invariant measure and the actions are orbit equivalent, then $G$ and $G^{\prime}$ are locally isomorphic.

In particular, we have:

THEOREM C. For $n \geqslant 2$, the actions of $\operatorname{SL}(n, Z)$ by automorphisms on $\mathbf{R}^{n} / Z^{n}$ are mutually nonorbit equivalent.

As with the standard rigidity theorems, Theorem A can be given a more geometric formulation. Namely, if we let $K$ be a maximal compact subgroup of $G$ and $S$ an essentially free ergodic $G$-space, then $S / K$ has the structure of a "Riemannian measurable foliation", i.e., a measure space with an equivalence relation such that each leaf (i.e., equivalence class) has the structure of a Riemannian manifold, and the assignment of Riemannian metric varies measurably over the entire measure space [10]. In the case of $S / K$, almost every leaf is of course just a symmetric space $G / K$.

THEOREM D. Let $F, F^{\prime}$ be Riemannian measurable foliations by symmetric spaces $G / K$ and $G^{\prime} / K^{\prime}$, where $G$ and $G^{\prime}$ are connected semisimple Lie groups with finite center and no compact factors. Suppose the foliations come from essentially free irreducible ergodic actions with finite invariant measure of $G$ and $G^{\prime}$ respectively, and that $G / K$ has rank at least 2 . Then if the foliations are transversally equivalent (i.e., the ergodic equivalence relations defined on suitable transversals are isomorphic), then $f$ and $F^{\prime}$ are isometric, modulo normalizing scalar multiples independent of the leaves, as Riemannian measurable foliations.

Thus, Theorem $D$ is asserting that for suitable $F$, the symmetric Riemannian structure on the leaves is completely determined by a purely measure theoretic invariant, namely the ergodic equivalence relation defined on a transversal.

The idea of the proof of Theorem $A$ is to combine the techniques used by Margulis in proving arithmeticity of lattices [5] with techniques and results from the cohomology theory of ergodic group actions, in particular, the notion of an amenable ergodic action [8], [9]. Our use of Margulis' techniques accounts for the assumption throughout that the real rank of $G$ be at least 2. The algebraic group nature of semisimple Lie groups is also used in fundamental ways throughout the proof. 
Details of the proofs will appear elsewhere. The author wishes to thank A. Connes and C. C. Moore for conversations concerning questions related to this work.

\section{REFERENCES}

1. A. Connes, J. Feldman and B. Weiss (to appear).

2. H. A. Dye, On groups of measure preserving transformations. I, Amer. J. Math. 8 (1959), $119-159$.

3. G. W. Mackey, Ergodic theory and virtual groups, Math. Ann. 166 (1966), $187-$ 207.

4. G. A. Margulis, Non-uniform lattices in semisimple algebraic groups, Lie Groups and Their Representations, (ed. I. M. Gelfand), Wiley, New York.

5. - Discrete groups of motions of manifolds of nonpositive curvature, Amer. Math. Soc. Trans., vol. 109, 1977, pp. 33-45.

6. G. D. Mostow, Strong rigidity of locally symmetric spaces, Ann. of Math. Studies, no. 78, Princeton Univ. Press, Princeton, N. J., 1973.

7. D. Ornstein and B. Weiss (to appear).

8. R. J. Zimmer, Amenable ergodic group actions and an application to Poisson boundaries of random walks, J. Functional Anal. 27 (1978), 350-372.

9. Induced and amenable ergodic actions of Lie groups, Ann. Sci. Ecole. Norm. Sup. 11 (1978), 407-428.

10. - Algebraic topology of ergodic Lie group actions and measurable foliations (preprint).

DEPARTMENT OF MATHEMATICS, UNIVERSITY OF CHICAGO, CHICAGO, ILLINOIS 60637 